\title{
CHRONIC INTUSSUSCEPTION IN INFANCY AND CHILDHOOD
}

\author{
BY \\ W. G. WYLLIE, M.D., F.R.C.P. \\ Physician, Hospital for Sick Children, Great Ormond Street, London \\ and \\ R. J. PUGH, M.B., M.R.C.P. \\ Late Registrar, Hospital for Sick Children, Great Ormond Street, London
}

It is often stated that chronic intussusception occurs mostly in older children, at about the seventh year. Three cases of this sort in boys aged seven, seven and a half, and eight years, have recently been recorded by Garvie and Kemp (1945), who appended a list of sixty-two previous case reports of chronic intussusception in which the ages of as many as thirty-three were in the first three years of life. In their case, aged seven and a half years, the history suggested that the onset of intussusception was at two and a half years of age. The term chronic is used to imply many days, weeks, or even years, without or until the occurrence of acute obstruction. An intermittent form can also be recognized.

A further three cases of chronic intussusception with unusual features in infants under three years of age are here described, and a fourth case, which appeared to undergo spontaneous reduction on two occasions, is also recorded.

Case 1. A male infant came under observation at the age of three months (November, 1944) and was subsequently admitted to hospital for investigation on four occasions.

History. The infant weighed $7 \mathrm{lb} .2 \mathrm{oz}$. at birth, but made slow progress in spite of breast feeding, weighing $8 \mathrm{lb}$. $3 \mathrm{oz}$. at three months. He was then brought to hospital because of loose stools with blood for three days and vomiting feeds for twentyfour hours. The abdomen was soft, not distended, and dehydration was not present. Vomiting gradually ceased in hospital and the stools became more satisfactory, though occasionally streaked with blood.

A possible intussusception was thought of, but rectally nothing was found; a barium enema was attempted with an indefinite result, and a barium meal appeared normal on follow-through by $x$ rays.

A mild degree of otitis media was present. The white blood cells were 10,500 per c.mm., of which 26 per cent. were polymorphs and 66 per cent. lymphocytes; Mantoux test and Wassermann reaction were negative. A month after admission the infant developed signs of pneumonia with rapid respirations and raised temperature, but made a good recovery on sulphadiazine. The infant took an adequate feed reluctantly and weighed only $8 \mathrm{lb}$. $12 \mathrm{oz}$ on discharge, aged five months.

The second admission was one month later for refusal of feeds and reappearance of abdominal pains. The stools were about three a day, yellow, and containing mucus. The weight was $9 \mathrm{lb} .3 \mathrm{oz}$. The abdomen looked distended, and, on palpation during a feed, a mass at the epigastric level was felt, which was of uniform consistency during palpation and was interpreted as a moderate hepatosplenomegaly. Under anaesthesia, the shape and consistency of the abdominal mass seemed to confirm the opinion of hepatic and.splenic enlargement. Various diagnoses were considered, but not confirmed, including von Gierke's disease in view of one initial very flat adrenaline-sugar curve. The infant gradually gained weight up to $10 \mathrm{lb}$. $5 \mathrm{oz}$. at eight months.

Under out-patient observation, from the age of eight to ten months, it was found that a steady but poor gain in weight occurred up to $12 \mathrm{lb}$., and bouts of abdominal pain were reported, but there was no irregularity of the stools except a tendency for these to be loose and offensive. The abdominal mass was felt on all occasions and thought to be increasing in size. Again, on the third admission to hospital, an $x$ ray and barium meal appeared to be normal.

The final admission was at eleven months, because of spasms of abdominal pain, frequent stools with occasional blood staining, and a temperature of $101 \cdot 2^{\circ} \mathrm{F}$. The white blood cells then numbered 17,000 per c.mm. The weight was $11 \mathrm{lb} .11 \mathrm{oz}$. On this occasion a barium meal outlined an obvious intussusception along the descending colon, but the infant collapsed and died before operation could be undertaken.

Post-mortem examination revealed a large intussusception involving the lower part of the ileum, caecum, and ascending and transverse colons invaginated into the descending colon. The duration of symptoms was eight months, with gradual increase in size of the abdominal mass, which at all examinations was never felt to contract or alter its shape.

Case 2. A girl, aged two years six months, was 
admitted to hospital with a history of vomiting for ten days, with abdominal pain, marked anorexia, and constipation. There had been no blood in the stools. The previous health of the patient had been satisfactory.

Examination revealed a flushed, irritable child, rather poorly nourished, whose temperature was $99.8^{\circ} \mathrm{F}$. and weight $26 \mathrm{lb}$.; the abdomen was tense and distended, making accurate palpation difficult and apparently painful. Rectal examination revealed no abnormality, and the other systems appeared normal.

A Mantoux test 1/1,000 was negative, the blood sedimentation rate raised to $18 \mathrm{~mm}$. in one hour, and $x$ ray of the chest and abdomen reported normal.

The abdominal pain continued intermittently, and guarding was noted in the epigastrium. Three days after admission, a blood-stained constipated stool was passed and it was thought that something could be felt on rectal examination. A laparotomy was performed by Mr. Twistington Higgins, which revealed an intussusception with an apex of distal ileum down to the pelvi-rectal junction. Manual reduction was effected except for an irreducible three inches of ileo-ileal intussusception which required resection. The proximal gut was exteriorized with a Paul's tube, and the distal portion by tying in a de Pezza catheter.

The immediate postoperative period was precarious, but was survived by means of intravenous fluids and duodenal suction. A second operation, consisting of a side-to-side anastomosis of the terminal ileum, was performed one week later. The patient's general condition necessitated liberal intravenous infusions of reconstituted plasma, blood, and glucose saline, for the succeeding ten days. Eventual recovery was complete five weeks after the first laparotomy and subsequent progress has been uneventful. Duration of intussusception was about thirteen days.

Case 3. A girl aged two years two months was admitted, in August, 1945, for investigation of a long and vague history of ill health. The patient was an only child, born weighing $5 \mathrm{lb} .7 \mathrm{oz}$; she thrived well on breast feeding until ten months, when for a period of one month she passed a great deal of unchanged blood in her stools, had frequent vomits, refused feeds, and lost weight rapidly.

A rectal swab at the onset of the diarrhoea provided a growth of Sonne dysentery B., and the patient was isolated at a fever hospital. After four weeks, improvement had taken place, but the diarrhoea and vomiting recurred ten days after her return home, still with streaks of blood in the stools. No further intestinal pathogens were discovered.

On first attendance at the Hospital for Sick Children, at the age of fifteen months, a history was given of bouts of abdominal pain, usually of daily occurrence but absent for two periods of four weeks each. The pains were associated with frequent, loose, pale stools without blood staining. At this time the patient weighed only $15 \mathrm{lb}$. $10 \mathrm{oz}$., and was paleand hypotonic; the liver was readily palpable, but no evidence of organic disease in the abdomen or elsewhere was elicited. Her tuberculin tests were negative and $x$ ray of the chest showed clear lung fields. When seen later after a period of two months she seemed much improved.

With a recurrence of intermittent abdominal colic and occasional vomiting in May and June, 1945, she was admitted to the Princess Beatrice Hospital where she was under observation for eight weeks. During this period no abdominal mass was palpated and no blood reported in the stools. In July, at the age of two years, the patient weighed only $17 \mathrm{lb} .3 \mathrm{oz}$., and difficulty was encountered in getting the child to take a solid diet, but on two occasions on examination in the out-patient department, no abnormal physical signs were found.

A final attendance in August with a recurrence of diarrhoea and vomiting, this time with blood in the stools, procured admission. Examination now revealed a sunken-eyed child, very poorly nourished, with a thickly coated tongue. The abdomen was slightly distended, while in the epigastrium a large mass, apparently in the colonic splenic flexure, was palpable. This was of rather resilient consistency, but did not alter in size after attempted colonic washouts. The rectum was ballooned and empty, while no spontaneous bowel actions were effected for three days, the lavage return being dark and containing much mucus. A barium enema was attempted but not retained.

The patient was examined under anaesthesia by Mr. Alan Small and a small lump was palpated in the left iliac fossa, of a soft consistency, and thought possibly to be an intussusception. The following day an exploratory laparotomy was performed after preparatory measures with intravenous fluids. Operation revealed a large intussusception of the terminal ileum extending as far as the sigmoid colon, with old adhesions around the entrance, and early patchy gangrene in the ascending colon. The intussusception was reducible as far as the junction of the middle and distal thirds of the transverse colon, where progress stopped and the bowel was seen to be of doubtful viability. A resection of gut was required and a side-to-side anastomosis performed.

The postoperative period was initially fairly satisfactory, aided by intravenous glucose saline, blood and plasma, but after five days signs of peritonitis were apparent. The patient's general condition rapidly deteriorated, death taking place suddenly seven days after operation.

Post-mortem examination showed that the distal stump of the transverse colon was gangrenous. There was patchy liver necrosis. Duration of the intussusception was probably one year and four months.

Case 4. A fourth case is reported in which spontaneous reduction appeared to occur on two occasions. A healthy male infant, of five and a half 
months, suddenly refused feeds, had a screaming attack with legs doubled up, and vomited several times. When the infant was first examined, there was a mass palpable on the left flank. The infant was pale, apathetic, with slightly sunken eyes. On admission to hospital the same day, no mass was palpable, the infant had.improved greatly in its general condition, and a saline enema produced a fairly good faecal action without blood. On the second day after admission, however, a further attack of screaming and vomiting occurred, and again a mass could be felt in the left half of the abdomen. This persisted only during the attack of pain and has apparently not returned. The stools at no time contained blood, and a barium meal and follow-through revealed no abnormality. The child has remained in good health and at the age of twelve months has had no further trouble.

\section{Discussion}

It is probable that, for an intussusception to become chronic, there must persist an abnormal or primitive type of mesenteric formation (Waugh, 1911). In place of the usual fusion of the peritoneum covering the ascending and descending portions of colon with the peritoneum of the posterior abdominal wall, there is a persistence of an ascending and descending mesocolon, and the longer these abnormal mesenteries are, the less tension there will be on the intussuscipiens and its blood supply. The gut remains viable, and congestion and bleeding are minimized until such times as increased size of the intussusception ultimately leads to signs of obstruction. The pull of the gut loaded with intussusception upon the splanchnic and renal plexuses can be provocative, Waugh stated, either of vomiting, or of pain referred to the hypogastrium and to the tip of the penis.

Sutherland (1932) observed that the passage of blood and mucus per rectum, and signs of intestinal obstruction, are often absent in cases of chronic intussusception. Of a total of sixty-nine case histories some degree of melaena on one or more occasions was present in thirty-two, rather less than half the total. Miller (1932) recorded a case of the intermittent type in a girl of three years. An abdominal tumour was present, which produced at no time any urgent symptom, and ultimately disappeared either spontaneously or aided by the bowel washouts which were given almost daily. Even with the tumour in existence, an opaque meal was almost entirely evacuated within twenty-four hours. Ten cases described by Dun (1923) illustrate the prolonged viability of the gut; symptoms nere present from four weeks to two and a half years, yet at operation all were readily reducible, without adhesions, and with recovery.

Our impression is that an X-ray film of a barium enema in infants and small children in the diagnosis of chronic intussusception is seldom helpful. Screening is essential, with the buttocks held closely together to prevent escape of the enema. An opaque meal may pass fairly readily along an intussusception, until such time as its size and state of congestion produce obstruction, when the picture becomes obvious. Still (1921) recorded a case in an infant of fourteen months with duration of symptoms for six weeks, where a bismuth meal and $x$ rays gave no assistance. Palpation under anaesthesia may be helpful, but the relaxation not only affects the muscles of the abdominal wall, but also that of the gut, whereby spasm producing a firm tumour is likely to be reduced (see case 3 , compare a small soft lump felt in the left iliac fossa under anaesthesia with the extensive intussusception found at operation the following day).

The vague onset of the chronic type makes the presence of intussusception unsuspected, as 90 per cent. of the cases seen in infancy are acute (Holt and McIntosh, 1940). The symptoms are most often confused with tuberculous peritonitis and other glandular causes of intestinal colic. Repeated clinical examination is essential in order to feel a tumour, yet, in the cases we have recorded, numerous examinations were made by several observers before the nature of the illness was suspected. Laparotomy may be necessary to confirm suspicions. There can be no doubt that intermittency and spontaneous reduction of an intussusception can occur. Garvie and Kemp (1945) mention that in a series of sixty-two recorded cases a tumour was felt at some time in only forty-seven. Most importance should be attached to an analysis of the case history, the frequent repetition of short attacks of abdominal cramp, it may be over weeks, often making the child cry or double up, the poor gain, or actual loss of weight, and in many instances the presence of a negative Mantoux. Watching the progress of a barium enema by screening is the most helpful form of radiography to employ.

Though the chronic type is uncommon, the accompanying graph is intended to show the frequency with which it occurs in infancy and early childhood.

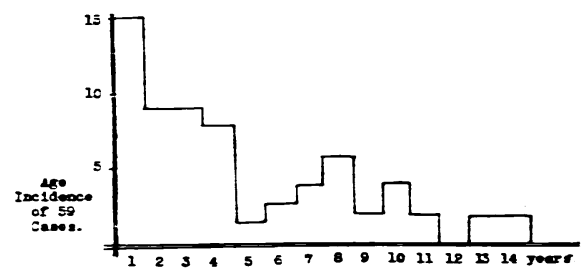

Age incidence of 59 cases of chronic intussusception.

\section{REFERENCES}

Dun, R. C. (1923). Brit. med.J., 1, 107.

Garvie, J. M., and Kemp, F. H. (1945). Arch. Dis. Childh., $20,73$.

Holt, L. E. and McIntosh, R. (1940). Diseases of Infancy and Childhood, New York.

Miller, R. (1932). Arch. Dis Childh., 7, 209.

Still, G. F. (1921). Arch. Paediat., 38, 174.

Sutherland, D. M. (1932). Arch. Dis. Childh., 7, 191.

Waugh, G.E. (1911). Lancet, 1, 1,492. 


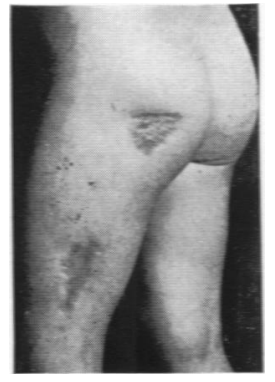

Fig. 1.-Scars on left buttock and thigh of case 1, two years after illness.

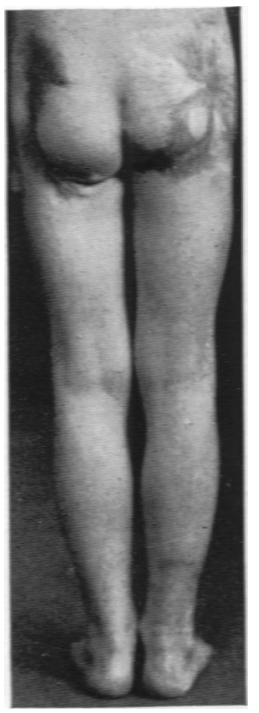

Fig. 4.-Scars on case 2: both buttocks and right ankle.

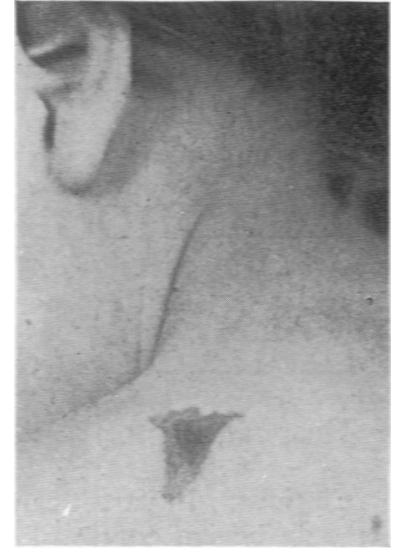

Fig. 2.-Isolated purpuric lesion on the shoulder of case 2.

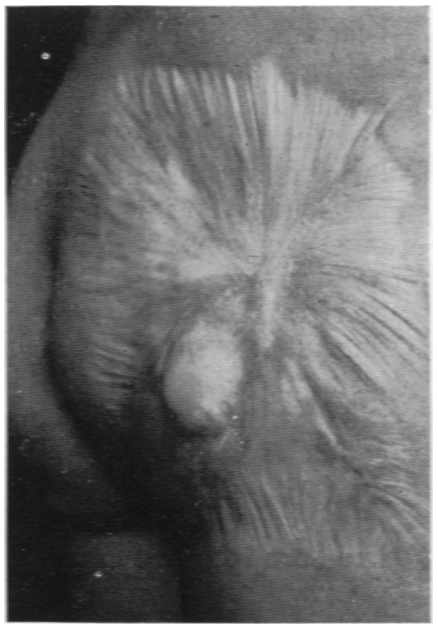

Fig. 5.-Scar on right buttock of case 2, taken three years after illness.

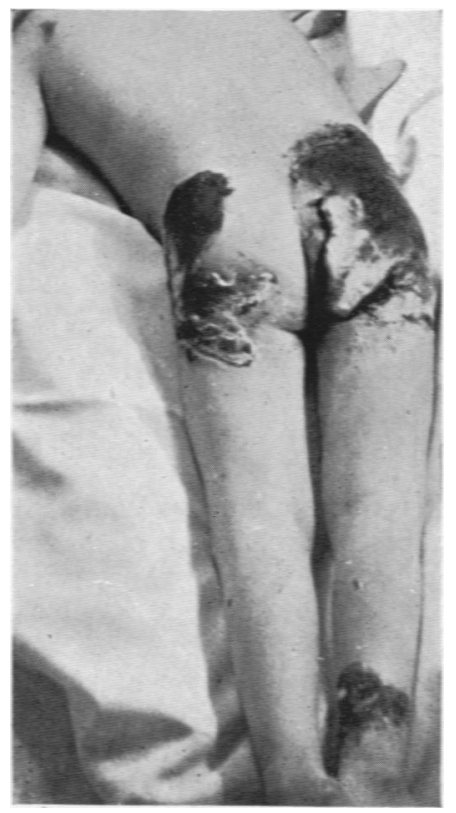

Fig. 3.-Stage of separation of the sloughs, case 2.

\section{BERI-BERI IN SINGAPORE $B Y$ G. HARIDAS}
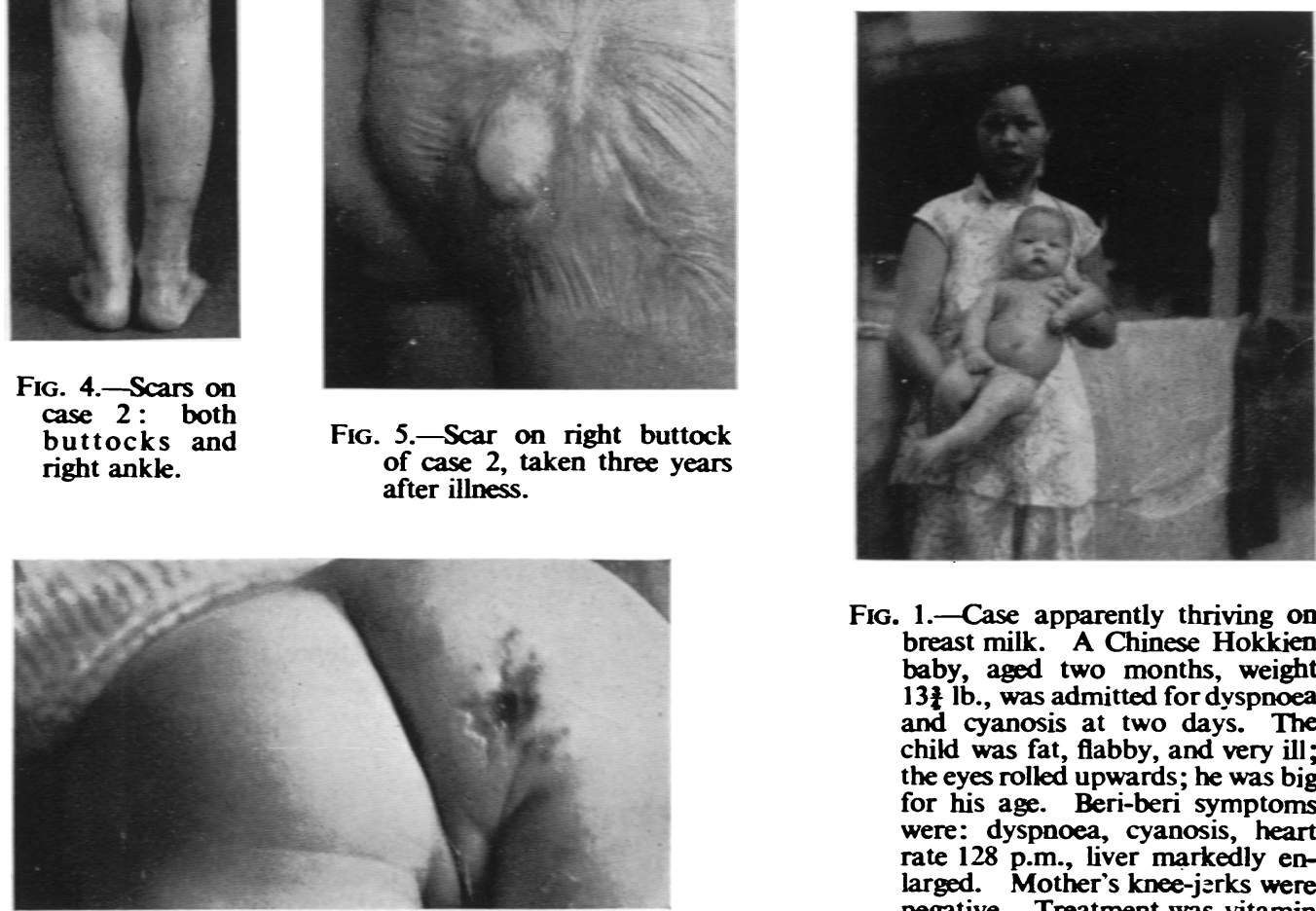

Fig. 6.-Scar on buttock of case 3.

C

Fig. 1.-Case apparently thriving on breast milk. A Chinese Hokkien baby, aged two months, weight 13: lb., was admitted for dyspnoea and cyanosis at two days. The child was fat, flabby, and very ill; the eyes rolled upwards; he was big for his age. Beri-beri symptoms were: dyspnoea, cyanosis, heart rate 128 p.m., liver markedly enlarged. Mother's knee-jerks were negative. Treatment was vitamin $B_{1}$, intramuscularly $15 \mathrm{mg}$. ; vitamin $B_{1}$ tablets by mouth. Photo taken after treatment. 